\title{
Notas sobre los molinos de harina de la dehesa de Zacatena en la Edad Moderna
}

\section{Francisco J. Moreno Díaz del Campo ${ }^{a}$, Francisco Fernández Izquierdo ${ }^{b}$ y Miguel F. Gómez Vozmediano ${ }^{c}$}

${ }^{a}$ Universidad de Castilla-La Mancha, Avda. de Camilo José Cela s/n, 13071 Ciudad Real. franciscoj.moreno@uclm.es, ${ }^{b}$ CCHS, Consejo Superior de Investigaciones Científicas, c/ Albasanz, 26-28, 28037 Madrid. francisco.fizquierdo@cchs.csic.es, ${ }^{\mathrm{c} A r c h i v o}$ Histórico de la Nobleza, c/ Duque de Lerma, 2, 45003 Toledo.miguel.gomez@cultura.gob.es.

\section{Resumen}

En la Edad Moderna, la dehesa de Zacatena (cuyo espacio, en la actualidad, ocupa en parte el Parque Nacional de Las Tablas de Daimiel) constituía una rica fuente de ingresos para la Corona, que, desde principios del siglo XVI, había asumido su gestión una vez que fueron incorporados los maestrazgos de las órdenes militares.

La dehesa acogía una flora y fauna de extremada riqueza y fue utilizada por los vecinos para hacer acopio de leña o para cazar y pescar. Gran parte de su razón de ser se debía a la enorme riqueza de sus pastos, pero Zacatena presentaba una peculiaridad: el elemento agua.

Debido a ello, en su área de influencia se construyeron y emplazaron infraestructuras arquitectónicas empleadas para aprovechar los recursos hídricos de los rios Guadiana y Cigüela, en especial molinos hidráulicos, muchos de los cuales datan de época musulmana.

La documentación histórica permite seguir la evolución de tales construcciones. A través de la misma, los autores persiguen mostrar la relevancia de la fuente escrita como complemento al trabajo de campo a la hora de reconstruir y conocer el patrimonio arquitectónico/arqueológico del enclave.

Palabras clave: patrimonio arquitectónico, agua, molinos hidráulicos, Zacatena, río Guadiana, siglos $X V-X I X$.

\begin{abstract}
During the Early Modern Times, Zacatena Medow (nowadays Las Tablas de Daimiel National Park) provided a lot of income to the Hispanic Monarchy, its owner since the early 16th century.

The area had a very rich flora and fauna used by locals to collect firewood or to hunt and fish. In fact, Zacatena existed for centuries thanks to its pastures, but the Medow had another peculiarity: water.

Due to this, in its area of influence, architectural infrastructures were built and located that were used to take advantage of the water resources of the Guadiana and Cigüela rivers. Among those constructions (many of which date back to the Muslim era) water mills were especially important. Historical documentation allows us to follow the evolution of these buildings. The authors intend to show the relevance of the written sources as a complement to the field work in order to reconstruct and know the architectural heritage of the place.
\end{abstract}

Keywords: architectural heritage, water, water mills, Zacatena, Guadiana River, 15th-19th centuries. 


\section{Introducción}

En la Edad Moderna, la dehesa de Zacatena (cuyo espacio, en la actualidad, ocupa en parte el Parque Nacional de Las Tablas de Daimiel, en la provincia de Ciudad Real) fue un referente económico para los pueblos comarcanos y constituía una rica fuente de ingresos para la Corona, que, desde principios del siglo XVI, había asumido su gestión una vez que fueron incorporados los maestrazgos de las órdenes militares.

Con una extensión cercana a las diez mil hectáreas, la dehesa acogía un ecosistema de una gran biodiversidad que fue explotado sistemáticamente tanto por la Real Hacienda en los pastos para ganado ovino y para la obtención de leña, con talas masivas en algunos momentos, o para cazar y pescar, incluso contraviniendo las normas que regulaban (y limitaban) dicha actividad. Gran parte de su razón de ser se debía a la enorme riqueza de sus pastos, pero Zacatena presentaba una peculiaridad: el elemento agua. Aproximadamente dos tercios de su extensión estaban formados por superficie encharcada, al quedar atravesada la dehesa por la confluencia de los ríos Cigüela y Guadiana, lo que proporcionó al enclave unas características muy definidas y una potencialidad aneja (Fig. 1). Con el correr de los siglos, en su área de influencia se emplazaron sucesivas construcciones empleadas para aprovechar la fuerza del agua en ambos ríos, que contribuyeron al mantenimiento de un medio lacustre, gracias a las presas artificiales que jalonaban el curso de los ríos para conseguir pequeños saltos de agua donde se situaban molinos escalonados.

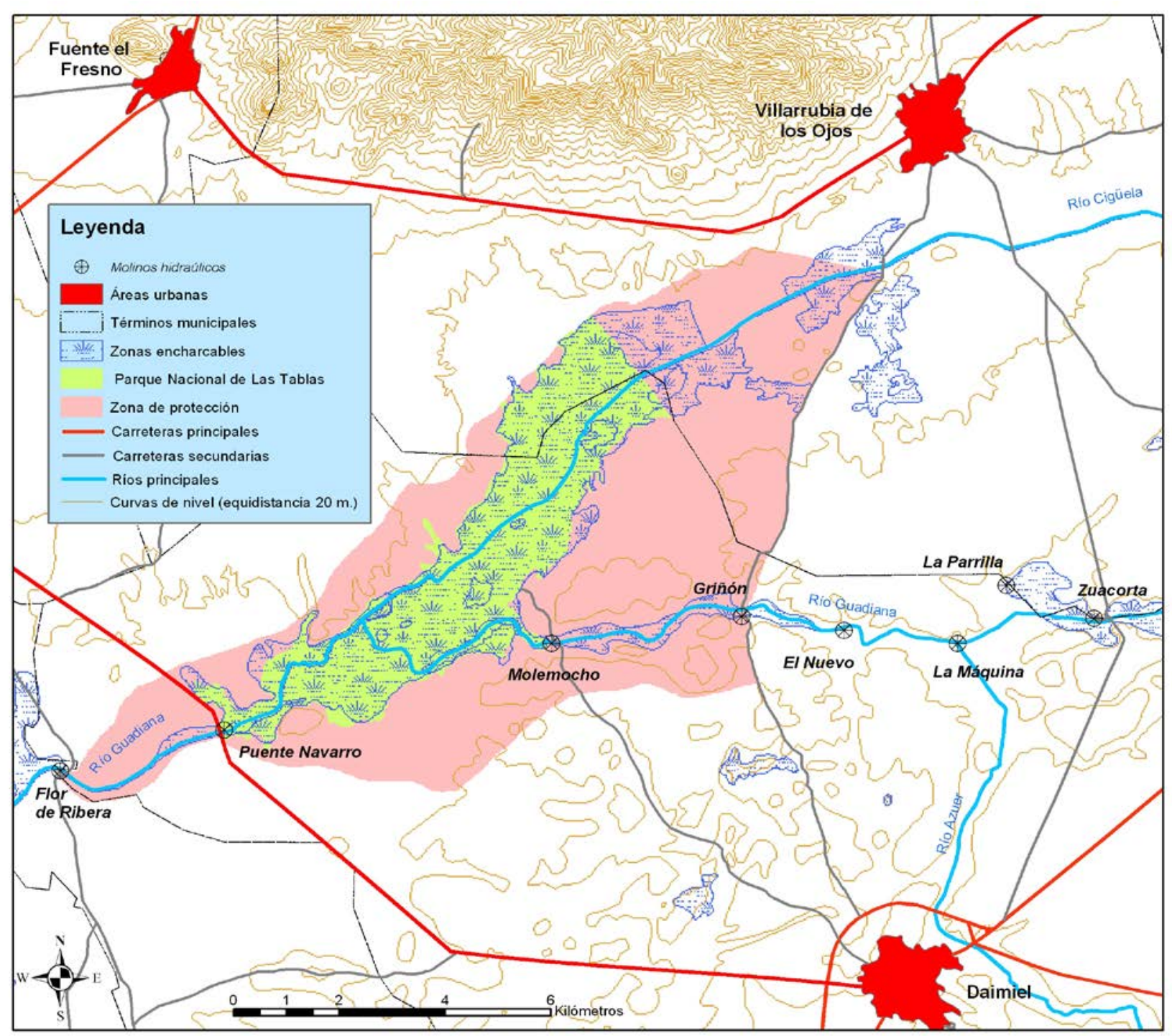

Fuente: Santos y Moreno (2009)

Fig. 1 Mapa de localización del actual Parque Nacional de Las Tablas de Daimiel y molinos harineros situados en su entorno 


\section{Los molinos de la dehesa}

En el área de influencia de Zacatena (dehesa propiamente dicha y tierras colindantes) tuvieron cabida casi una decena de molinos cuya capacidad de molienda hizo que la zona se convirtiera en foco de atracción para comerciantes y agricultores de las comarcas limítrofes. Ya lo decía un viejo adagio de la zona: "el camino del Guadiana, que el que lo anda, lo gana".

Junto a los molinos, el conjunto de construcciones hidráulicas se completa con los batanes, los puentes y las presas. Los restantes edificios de la dehesa eran humildes edificaciones de diverso tipo destinadas a satisfacer las necesidades de quienes vivían y trabajaban en sus alrededores: chozos, majadas y corrales de ganado, caleras, "casillas" de pescadores, más algunas casas de labor y guardería, que completaban los edificios de la dehesa.

Se estima que determinadas construcciones datan de época musulmana, en especial algunos de los molinos hidráulicos más importantes y rentables como Molemocho (previamente conocido como Nuño Mocho) y Griñón, acaso los más conocidos. Con todo, el conjunto de molinos en Zacatena no fue estático y permaneció en constante cambio, adaptándose a las necesidades económicas y productivas de cada momento, además de al desgaste y riesgo de ruina en los temporales y avenidas (como la de 1545), pese a que los caudales en esta zona de la cuenca alta del Guadiana no fueron excesivos.

La importancia de los molinos fluviales y de los batanes en una región como la del Guadiana durante el Antiguo Régimen, se deriva de tres cuestiones (Gómez Vozmediano, 1999). En primer lugar, por su importancia económica, ya que la molturación de grano resultaba imprescindible para abastecer de harina a la población del entorno. Asimismo, los batanes atendían la producción de pañería local, especialmente a partir de lanas, pero con un rendimiento mucho menos interesante económicamente que la molienda de granos. Cuando aumentó la población en las llanuras manchegas, ante la escasez de recursos hidráulicos para la molienda, se fomentó la construcción de molinos de viento, traídos por la Orden de San Juan de sus instalaciones en la isla de Rodas y Malta, que carecían de corrientes fluviales (Almarcha, 2005; Porras, 2016). Junto a ello, debe señalarse que los molinos eran instalaciones rentables para la inversión, sin que estuvieran sometidos en su propiedad a monopolios feudales, aunque necesitaban de una licencia regia para construirlos y no perjudicar a los ya existentes, como ya regulaban las Partidas.

La Orden de Calatrava, como titular del señorío territorial, se ocupó de su construcción desde la baja Edad Media, y exigía a la población del Campo de Calatrava acudir obligatoriamente a sus molinos, pero no pudo impedir la competencia de otros nuevos levantados por particulares o la pérdida de los existentes, que en tiempo del emperador Carlos V pasaron a los compradores de encomiendas a las que pertenecían. Finalmente no debe olvidarse su papel como articuladores del territorio. La red de caminos que atravesaba la comarca y conectaba unas localidades con otras lo hacían tomando como referencia el río y los ingenios que lo jalonaban (Moreno, 2014).

Durante el Antiguo Régimen, la propiedad de los molinos harineros con parada en Zacatena correspondió a diferentes instancias, bien fueran particulares, bien institucionales. La Orden de Calatrava era propietaria de los instalados desde su implantación en el siglo XII, y a la altura del siglo XV era dueña casi exclusiva de los molinos en este sector del Guadiana. Gran parte de los mismos seguía bajo su dominio en 1567, cuando el Consejo de las Órdenes ordenó amojonar de nuevo sus límites (Tabla 1). De aquel pormenorizado apeo datan algunas de las referencias que permiten acercarse al origen de estos ingenios, de ahí su valor. También conocer a sus dueños y constatar-mediante el cruce con otras fuentes- que, entre los siglos XVI y XVIII, comenzaron a intervenir particulares, tanto por compra a la orden en las encomiendas enajenadas (Villarrubia, Malagón, Fuentelemperador), como con nuevas concesiones (Gaspar Rótulo, milanés factor del arrendamiento de la mesa maestral de Calatrava y negociante de la carrera de Indias ${ }^{1}$, o Hernando de Castro $^{2}$ ).

\footnotetext{
${ }^{1}$ Gaspar Rótulo, vecino de Almagro, dueño de la nao 'La Trinidad', reclama la indemnización debida por habérsele embargado dicha embarcación para la armada de Blasco Núñez Vela, y que se perdió en la Bahía de Cádiz (1539-1541). AGI. Justicia, leg, 1171, nº. 7. En 1550, junto a su esposa María Carrillo Osorio, fundan mayorazgo a favor de su hijo Galasso Rotulo, vinculando unas casas en Almagro (Ciudad Real), así como la tierra y jurisdicción de las villas de Fines y Somontín (Almería); curiosamente este documento está trufado de símbolos y dibujos cinegéticos (halcones, podencos persiguiendo a liebres, etc.). ARCHV, Pergaminos, caja 79, exp. 3. Ávido inversor en juros, termina arrendando alumbres y tiene tratos con
} 
Tabla 1. Los molinos de Zacatena según el amojonamiento de 1567

\begin{tabular}{|c|c|c|c|c|c|c|}
\hline \multirow[t]{2}{*}{ Nombre } & \multirow[t]{2}{*}{ Ubicación } & \multicolumn{3}{|c|}{$\begin{array}{c}\text { Citados en el } \\
\text { amojonamiento y } \\
\text { número de ruedas }\end{array}$} & \multirow[t]{2}{*}{ Propietarios } & \multirow[t]{2}{*}{$\begin{array}{c}\text { Estado de } \\
\text { conservación }\end{array}$} \\
\hline & & $1433^{1}$ & $1550-2^{2}$ & $1567^{3}$ & & \\
\hline $\begin{array}{l}\text { El Arquel } \\
\text { (Alcorquel) }^{4}\end{array}$ & $\begin{array}{l}03^{\circ} 32^{\prime} 47^{\prime \prime} \mathrm{W} \\
39^{\circ} 08^{\prime} 05^{\prime \prime} \mathrm{N}\end{array}$ & & & $\mathrm{x}$ & & desaparecido \\
\hline $\begin{array}{l}\text { Zuacorta } \\
\text { (Azuda Corta, } \\
\text { Zudacorta) }\end{array}$ & $\begin{array}{l}03^{\circ} 34^{\prime} 11^{\prime \prime} \mathrm{W} \\
39^{\circ} 08^{\prime} 02^{\prime \prime} \mathrm{N}\end{array}$ & & 2 & $\mathrm{x}$ & $\begin{array}{l}\text { Convento de Sta. } \\
\mathrm{M}^{\mathrm{a}} \text { del Monte (San } \\
\text { Juan) }\end{array}$ & restaurado \\
\hline La Parrilla $^{5}$ & $\begin{array}{l}03^{\circ} 35^{\prime} 27^{\prime \prime} \mathrm{W} \\
39^{\circ} 08^{\prime} 20^{\prime \prime} \mathrm{N}\end{array}$ & $\mathrm{x}, 2$ & 2 & $\mathrm{x}$ & $\begin{array}{l}\text { Convento de } \\
\text { Calatrava }\end{array}$ & $\begin{array}{l}\text { ruinoso; restos } \\
\text { parciales }\end{array}$ \\
\hline La Dehesa $^{6}$ & $\begin{array}{l}03^{\circ} 36^{\prime} 03^{\prime \prime} \mathrm{W} \\
39^{\circ} 07^{\prime} 44^{\prime \prime} \mathrm{N}\end{array}$ & $x$ & 2 & $\mathrm{x}$ & $\begin{array}{ll}\text { Encomienda } & \text { de } \\
\text { Daimiel } & \\
\text { Encomienda } & \text { de }\end{array}$ & desaparecido \\
\hline $\begin{array}{l}\text { El Nuevo de } \\
\text { Curenga }^{7}\end{array}$ & $\begin{array}{l}03^{\circ} 37^{\prime} 39^{\prime \prime} \mathrm{W} \\
39^{\circ} 07^{\prime} 51^{\prime \prime} \mathrm{N}\end{array}$ & & 4 & $\mathrm{x}$ & $\begin{array}{l}\text { Daimiel }(50 \%) \text { y } \\
\text { frailes de Almagro } \\
(50 \%)\end{array}$ & $\begin{array}{ll}\text { ruinoso; } & \text { restos } \\
\text { parciales } & \end{array}$ \\
\hline Griñón & $\begin{array}{l}03^{\circ} 38^{\prime} 56^{\prime \prime} \mathrm{W} \\
39^{\circ} 08^{\prime} 00^{\prime \prime} \mathrm{N}\end{array}$ & $\mathrm{x}$ & 5 & $\mathrm{x}$ & $\begin{array}{l}\text { Enc. Villarrubia } \\
\text { (desmembrada) }\end{array}$ & $\begin{array}{l}\text { ruinoso; restos } \\
\text { parciales }\end{array}$ \\
\hline $\begin{array}{l}\text { Molemocho } \\
\text { (Muño Mocho) }^{8}\end{array}$ & $\begin{array}{l}03^{\circ} 41^{\prime} 30^{\prime \prime} \mathrm{W} \\
39^{\circ} 07^{\prime} 44^{\prime \prime} \mathrm{N}\end{array}$ & $\mathrm{x}$ & 4 & $\mathrm{x}$ & $\begin{array}{l}\text { Mesa maestral de } \\
\text { Calatrava }\end{array}$ & restaurado \\
\hline $\begin{array}{l}\text { El de Gaspar } \\
\text { (Gaspar } \\
\text { Rótulo) }\end{array}$ & $\begin{array}{l}03^{\circ} 45^{\prime} 03^{\prime \prime} \mathrm{W} \\
39^{\circ} 07^{\prime} 09^{\prime \prime} \mathrm{N}\end{array}$ & & 4 & $\mathrm{x}$ & Gaspar Rótulo & desaparecido \\
\hline El Navarro ${ }^{10}$ & $\begin{array}{l}03^{\circ} 45^{\prime} 47^{\prime \prime} \mathrm{W} \\
39^{\circ} 06^{\prime} 42^{\prime \prime} \mathrm{N}\end{array}$ & $\mathrm{x}$ & 4 & & $\begin{array}{l}\text { Encomienda de } \\
\text { Jetar-Daimiel }\end{array}$ & $\begin{array}{l}\text { parcialmente } \\
\text { restaurado }\end{array}$ \\
\hline Flor de Ribera ${ }^{11}$ & $\begin{array}{l}03^{\circ} 47^{\prime} 59^{\prime \prime} \mathrm{W} \\
39^{\circ} 06^{\prime} 18^{\prime \prime} \mathrm{N}\end{array}$ & & 2 & $\mathrm{x}$ & $\begin{array}{ll}\text { Mujer e hijos } & \text { de } \\
\text { Hernando } & \text { de } \\
\text { Castro } & \end{array}$ & $\begin{array}{l}\text { ruinoso; } \\
\text { parciales }\end{array}$ \\
\hline
\end{tabular}

Fuentes:

1. AHN, OOMM, Judicial, leg. 38285.

2. AHN, OOMM, Judicial, legs. 39448 y 40967. El fiscal de Calatrava con Francisco de Benavides, comendador de Daimiel y guarda mayor de Zacatena, sobre cortas en la dehesa. Se anotan las ruedas de molinos y batanes en todas las instalaciones de la ribera del Guadiana con derecho a surtirse de madera en dicha dehesa para la reparación y conservación de mecanismos y edificios.

3. AHN, OOMM, Judicial, leg. 38285

4. La encomienda de Villarrubia poseía los molinos de Ahorquel o el Arquel, el Robledo, el Rieto y Griñón, estos dos últimos citados en los inventarios desde 1459 (Solano, 1976: 233 y 432; Corchado, 1983, 415)

5. El maestre García López de Padilla concedió medio molino de la Parrilla al Convento de Calatrava, que lo arrendaba para obtener su alquiler. En 1547 se adjudicó por tres años a Antón de Toledo y Fernando Gómez, por 37.500 maravedíes (100 ducados). AHN, OOMM, Judicial, leg. 38285.

6. En el mapa, llamado "La Máquina", denominación con la que es conocido en la actualidad

7. En el mapa, llamado "El Nuevo. El molino de Caruenga o Curuenga existía al menos desde 1459, junto con unos batanes, conforme a los primeros inentarios y visitas conservados de los bienes de la encomienda de Daimiel (Solano, 1976: 211 y 418) y AHN, OOMM, Judicial, leg. 38169.

8. Mencionado desde 1422 en visitas al Campo de Calatrava efectuadas por la orden, junto a otros inmuebles de la mesa maestral, Malvecino y Batanejo (Corchado, 1983: 28). En 1511 hay constancia de una intervención con cargo a rentas de la mesa maestral en los cuatro molinos de Nuño Mocho, Malvecino, la Celada y el Batanejo. AHN, OOMM, libro 323 C, f. 26 r.

9. Gaspar Rótulo, de origen milanés, participó con los Fugger en la gestión del arrendamiento del maestrazgo de Calatrava desde la segunda década del siglo XVI, estableciéndose en Almagro, donde llegaría a ser regidor perpetuo de su ayuntamiento. Este molino, situado en Calabazas, fue derribado en 1561 cumpliendo una sentencia del Consejo Real, a instancias del concejo de Villarrubia. AHN OOMM, Judicial, leg. 36744.

10. Recogido en los inventarios de bienes de la encomienda de Daimiel desde 1459

11. En 1533, en el transcurso de una investigación judicial sobre cortas en la dehesa de Zacatena, los testigos informaron que Hernando de Castro, mayordomo del comendador de Daimiel -que tenía también el cargo de guarda mayor de la dehesa de Zacatena-, con ayuda de su sobrino Gonzalo Martínez de Castro, estaban construyendo un molino en el Guadiana, aún sin nombre. AHN, OOMM, Judicial, 43856.

negociantes genoveses de Toledo y cambiadores de Medina del Campo. También fue regidor de Toledo e incluso procurador en Cortes por dicha ciudad, pretendiendo un hábito de órdenes.

${ }^{2}$ Los Castro son otra saga de negociantes, judeoconversos emparentados con los Gutierre de la Caballería. Negociaron con paños e hicieron préstamos, adquiriendo gran notoriedad en Almagro, de donde fueron regidores. 
La orden, que había tenido el monopolio de la molienda, solo mantuvo el dominio directo de la mitad de las muelas corrientes, desde mediados del siglo XVI y durante toda la Edad Moderna (Moreno, 2005, p. 2014). Con todo, es necesario puntualizar que ese control se repartió entre diferentes instituciones dentro de la propia orden. En el caso concreto de los molinos sitos en el entorno de Zacatena, cabe señalar hasta tres propietarios diferentes. En primer lugar, el Sacro Convento de Calatrava la Nueva, que controló la mitad del molino de La Parilla. En segundo término, la mesa maestral (léase la Real Hacienda), que gestionó el molino de Molemocho y, más allá de los límites de la dehesa, el de Malvecino y la mitad de La Celada, más un "batanejo" también situado fuera de la dehesa. Finalmente, la encomienda de Daimiel, a la que correspondió la gestión de los molinos de La Dehesa, el Navarro y de la mitad del Nuevo de Curenga, además de dos batanes, quedando los de Griñón, La Celada, El Emperador y la Torre, en manos privadas de los compradores de sus respectivas encomiendas.

\section{Los molinos, singularidad constructiva y particularidades técnicas}

Los molinos fluviales son unos hitos fundamentales en el paisaje agrario tradicional. Desde hace mucho tiempo inactivos, durante siglos concitaron tanto inversiones como un hormigueo de gentes y cereal por los caminos o senderos que confluían en ellos, siendo básicos en la economía agrícola y en el abasto alimenticio de hombres y animales domésticos.

En su inmensa mayoría fueron levantados por artífices o alamines locales, aunque, a raíz de lo observado en otros territorios, no debe descartarse la participación de "maestros de molinos" y de ingenieros, sobre todo en la edificación de aquellos que tuvieron un mayor tamaño o cuya construcción era especialmente compleja. Los ingenios del entorno de Zacatena (como los del resto del alto Guadiana y sus afluentes) (Gómez Vozmediano, 1999) respondieron a una misma estructura constructiva. Todos estaban dotados de sala de molienda, almacén de grano y algún poyo corrido para descansar o pernoctar. Su planta solía ser rectangular o cuadrada en algún caso. La construcción era sencilla, propia de un complejo industrial. Su cimentación, asentada sobre el lecho del propio río, se llevaba a cabo con grandes piedras y mortero. Las paredes, normalmente de tapia (aunque reforzadas con piedra), tenían pocos vanos y en su mayoría fueron ventanucos altos, que eran construidos así para evitar corrientes de aire que desperdigaran la harina. Solían encalarse por la parte de la fachada y el interior se daba una primera capa con estiércol de vaca, que, posteriormente, también era encalada. El suelo se empedraba si se construía en la orilla, pero se enlosaba con baldosas de barro cocido, si se edificaba sobre el cauce del río.

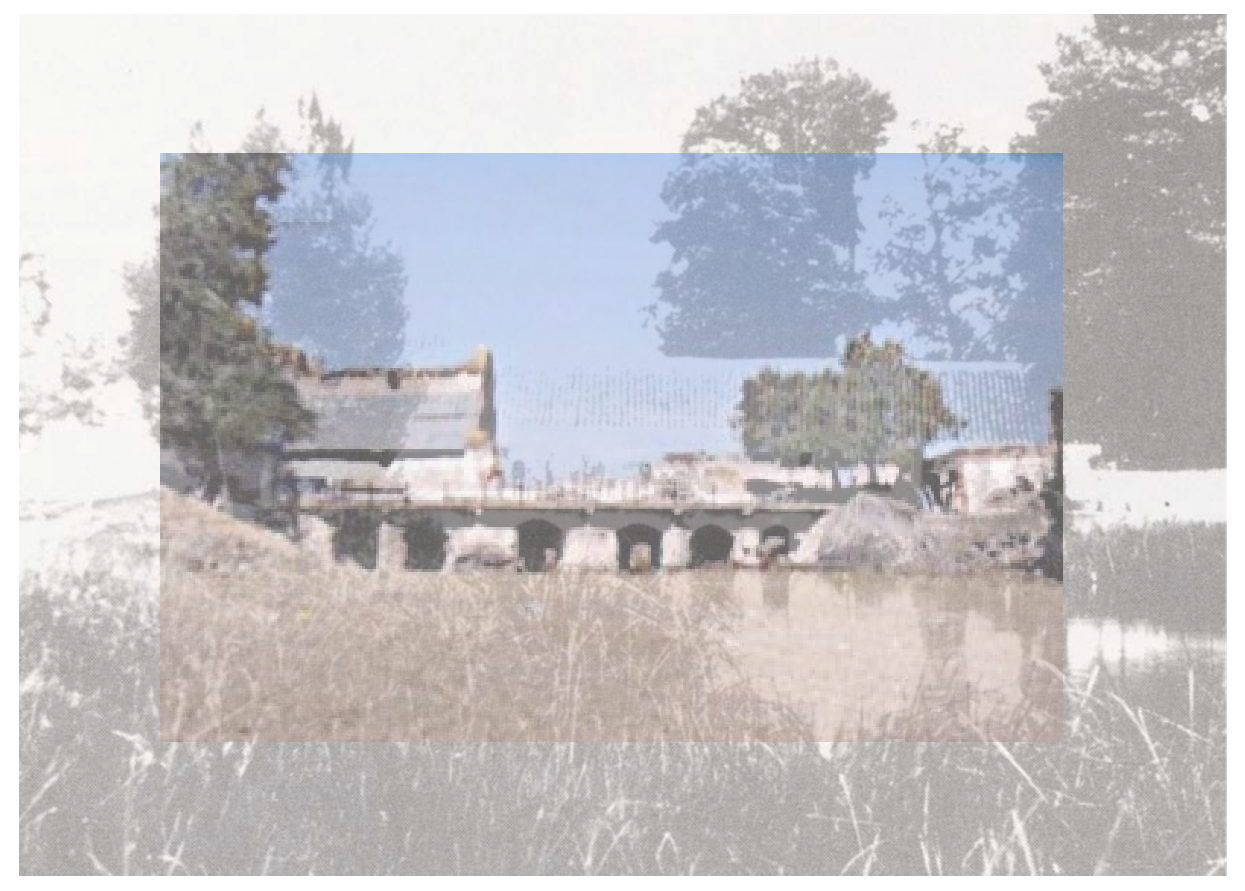

Fig. 2 El molino de Flor de Ribera. Composición fotográfica comparativa años 1960 (b/n) y 2018 (color) 
El tejado era de teja árabe, a dos aguas y vertiente suave y se sostenía sobre una armazón de vigas de madera, por lo general de pino o "rebollo" (roble), aunque la madera más abundante en el entorno era la de encina. A veces se hacía un cielo raso con juncos, carrizo o cañizo trabados con barro, para que no cayese polvo ni agua a la harina ni al grano.

En el caso del alto Guadiana, donde el desnivel es prácticamente nulo y la corriente de agua apenas si tiene fuerza para generar la puesta en marcha de la maquinaria de molienda, el exterior de los molinos se completaba con los denominados azudes, barreras levantadas en el cauce del río, generalmente construidas con piedra, mortero y tierra, cuya misión principal consistía en formar una balsa en la que almacenar agua, elevar su nivel y derivar la corriente hacia un salto que accionaba la maquinaria de molienda.

Las balsas de los molinos de Zacatena también sirvieron como "pesqueras", viveros de peces, cuya explotación era arrendada a terceros por los propietarios de los molinos, particularmente la mesa maestral de Calatrava y la encomienda de Daimiel. En el caso de los ingenios que nos ocupan, el azud también fue utilizado como puente para cruzar el río (Fig. 2), fuente de conflictos entre los propietarios y vecinos de los pueblos comarcanos (Moreno, 2005).

Tanto los edificios como la maquinaria de los molinos estaban muy expuestas al deterioro. La erosión generada por el agua, potenciada durante las avenidas puntuales y el propio uso de los azudes como lugares de paso convirtió a estas presas en objeto de atención preferente de los propietarios, dado que una deficiente conservación de las mismas podía generar pérdidas y roturas que provocaran un menoscabo en la producción del propio molino.

Dada su larga trayectoria histórica (algunos proceden de época musulmana) (Rodríguez-Picavea, 1996; Hervás, 2011), los molinos de Zacatena pasaron por diferentes estadios constructivos, cuestión que dificultad la datación de aquellos que se mantienen en pie o de los restos arquitectónicos que se conservan. Esa larga historia es la que ha propiciado que algunos de los ingenios a los que nos referimos hayan dispuesto de diferentes mecanismos de molienda a lo largo de su vida útil. De hecho, los más antiguos fueron ruedas verticales (aceñas), que aún funcionaban entre 1552 y 1557 en los molinos de Malvecino, la Celada y Gaspar Rótulo ${ }^{3}$, si bien lo más plausible es que la inmensa mayoría de ellos emplease ruedas horizontales, rodeznos, documentados en los mismo años en Nuño Mocho y el Batanejo, dispuestas en primer momento al descubierto y más tarde, a partir del siglo XVI en cubas de regolfo (Moreno, 2014). Los restos de algunos de estos molinos (Fig. 2) y los trabajos arqueológico-arquitectónicos paralelos a la restauración de otros (como por ejemplo Molemocho) (Almagro, 2009), confirman que este último sistema fue el predominante durante la Edad Moderna, acaso por ser el que mejores resultados proporcionaba a la hora de aprovechar las calmas aguas del Guadiana. La documentación histórica avala dicha afirmación y, además, informa del especial cuidado que los propietarios tenían en la conservación de los elementos estructurales como tajamares, cárcavas, cimientos, cubas... que por estar en contacto constante con el agua sufrían sus embates directos y estaban sometidos a un desgaste mayor que el resto de elementos constructivos tal y como prueban algunos de los proyectos de rehabilitación que tuvieron lugar en Zacatena en la propia Edad Moderna (Moreno, 2016).

Finalmente cabe hablar de las piedras de molino (muelas) con forma de rueda maciza, horadadas en el centro, para facilitar el acoplamiento a los mecanismos que las movían y para introducir los granos, y colocadas horizontalmente una sobre otra. Como es conocido, son de dos tipos: la solera fija (estriada con rayones oblicuos, líneas radiales excéntricas en forma de abanico para expulsar la harina), sobre la que se desplaza la corredera o volaera. Ambas se fabricaban con piedra dura: pedernal o granito (también llamado popularmente piedra morena), de gran peso, lo que facilitaba la trituración o mixtura del grano ${ }^{4}$. La piedra volcánica, muy frecuente en la zona, no era recomendable porque su arenisca se deshacía/erosionaba con facilidad. Las muelas más modernas se construyeron de cemento. De la cantera de piedras de molino de Ruidera se extrajeron durante siglos las muelas de los molinos y batanes comarcanos, aunque no debe descartarse que algunas de las empleadas en Zacatena procedieran del Campo de San Juan y más concretamente de la cantera de Piédrola (Cárdenas, 2018).

\footnotetext{
${ }^{3}$ De acuerdo con el destino de las licencias para obtener madera de la dehesa para reparar molinos, AHN, OOMM, Judicial, leg. 36744.

${ }^{4}$ El cereal, para ser molido sin quemarlo y no quedar reducido a polvo, sino facilitar que se tronzara para separarlo de la cascarilla, debía pasar rápidamente del interior de las muelas al exterior; es decir desde la entrada al mordiente. Para ello los molineros se ocupaban del repicado de las piedras molineras.
} 


\section{A modo de conclusión: la documentación de la Orden de Calatrava como base para la reconstrucción del patrimonio molinar de Zacatena}

La documentación histórica relativa a la dehesa, en su inmensa mayoría conservada en la sección de Órdenes Militares del Archivo Histórico Nacional, permite seguir la evolución arquitectónica de los molinos, conocer cuándo se construyeron, saber del alcance, significación y presupuesto de las obras acometidas para su mantenimiento y estar al tanto de qué materiales se emplearon en tales actuaciones.

Para profundizar en dichas cuestiones, se dispone, principalmente, de tres tipos de fuentes:

a) En primer lugar la gestión de la propiedad y control sobre la misma que realizaba la Orden de Calatrava, mediante visitas, inventarios y reconocimientos, tanto de los bienes pertenecientes a la mesa maestral, como a las encomiendas. En este grupo de fuentes, deben incluirse las que hacen referencia a las reparaciones que se gestionaban desde el Consejo de Órdenes con cargo a una partida de fondos que cada año se aprovisionaba desde las rentas maestrales, y las intervenciones de los gobernadores del partido de Almagro o comisionados enviados puntualmente desde la corte. Estos documentos se conservan en la sección de Órdenes Militares en el Archivo Histórico Nacional.

b) En segundo término, la gestión económica de los propios molinos, que en el caso de las encomiendas estaría en manos de sus comendadores o administradores, y en las mesas maestrales estaba incluida en los asientos de los maestrazgos de las órdenes militares, controlados por los Fugger la mayor parte del tiempo desde el reinado de Carlos I, cuya documentación se reparte entre el Archivo General de Simancas y los archivos de protocolos notariales. Asimismo, las encomiendas que se enajenaron en el siglo XVI fueron sometidas a inventarios y tasaciones, incluidos los molinos.

c) Finalmente, los numerosos litigios que se derivaban de los aprovechamientos hidráulicos y pesqueros tenían su acogida en los tribunales regios, el principal el Consejo de Órdenes, cuyos procesos se encuentran en el denominado Archivo de Toledo, en el AHN, y otros en la Real Chancillería de Granada, donde se recibían las apelaciones de los restantes territorios que no controlaba el consejo citado. Aparte de esta documentación, la mención a molinos se recoge en fuentes generales, como las Relaciones topográficas o las respuestas al Catastro de Ensenada (1752) y en inventarios y fuentes estadísticas posteriores de los siglos XIX y XX.

\section{Referencias}

Almagro García, N. (2009). Rehabilitación del molino de Molemocho (1998- 2000). En Actas del V Congreso Internacional de Molinología (Alcázar de San Juan, del 19 al 21 de octubre de 2005) (pp. 753-757). Toledo: Empresa Pública don Quijote de La Mancha.

Almarcha Núñez-Herrador, E., Barba Ruedas, C., y Peris Sánchez, D. (2005). Ingenios de agua y aire. Toledo: Empresa Pública don Quijote de La Mancha.

Cárdenas Martín-Buitrago, Á. J. (2018). Las canteras de Piédrola. Un ejemplo de producción molera en La Mancha (Trabajo Fin de Máster), Universidad de Castilla-La Mancha, Ciudad Real.

Corchado Soriano, M. (1983). Las jerarquías de la Orden con rentas en el Campo de Calatrava. Ciudad Real: Instituto de Estudios Manchegos.

Gómez Vozmediano, M. F. (1999). Los molinos de harina riberiegos en la comarca de Puertollano (siglos XIV-XX). Revista Campo de Calatrava, 1, 15-98.

Hervás Herrera, M. Á. (2011). Molinos hidráulicos harineros de ribera en el Alto Guadiana. De los Ojos de Villarrubia a El Emperador. En P. Serrano de Menchén (Coord.), Tierra de cultura. I Curso de Historia de Argamasilla de Alba y Alto Guadiana, (pp. 9-32). Argamasilla de Alba, España: Los Académicos de Argamasilla.

Melero Cabañas, D. (2014). Ciudad Real, tierra de molinos de agua, Ciudad Real: Excma. Diputación Provincial de Ciudad Real.

Moreno Díaz del Campo, F. J. (2005). El molino de Flor de Ribera y la disputa por el control de los recursos del río Guadiana a principios del siglo XVIII en Torralba de Calatrava. En M. Romero Fernández y F. Alía Miranda (Coords.), Historia de Torralba 
de Calatrava. Actas de las I y II Jornadas monográficas sobre Torralba de Calatrava y su entorno (2003-2004) (pp. 315-342). Torralba de Calatrava: Ayuntamiento de Torralba de Calatrava/Caja Rural Provincial de Ciudad Real.

Moreno Díaz del Campo, F. J. (2014). El aprovechamiento hidráulico del Guadiana: los molinos de ribera. Siglos XV-XIX. En M. Mejías Moreno (Ed.), Las Tablas y los Ojos del Guadiana: agua, paisaje y gente (pp. 147-84). Madrid: IGME-OAPN.

Moreno Díaz del Campo, F. J. (2016). Obras y reparos en el molino. La Orden de Calatrava y la gestión de sus infraestructuras industriales en la Edad Moderna. En E. Vicedo Rius (Coord.), Pagesia, indústria i món rural. VIIIè Congrés sobre Sistemes agraris, organització social i poder local (pp. 177-200). Lleida: Fundación Pública Institut d'Estudis Ilerdenses, Diputació de Lleida.

Porras Arboledas, P. A. (2016). Los molinos de viento de La Mancha santiaguista. El molino como sintoma y como símbolo. Campo de Criptana: Ayuntamiento de Campo de Criptana-Excma. Dip. Prov. de Ciudad Real.

Rodríguez-Picavea Matilla, E. (1994). La formación del feudalismo en la meseta meridional castellana: los señoríos de la Orden de Calatrava en los siglos XII-XIII. Madrid-México DF: Siglo XXI.

Rodríguez-Picavea Matilla, E. (1996). La difusión del molino hidráulico en el Campo de Calatrava (siglos XII-XIV). En R. Izquierdo Benito y F. Ruiz Gómez (Coords.), Alarcos, 1195. Actas del Congreso Internacional Conmemorativo del VII Centenario de la Batalla de Alarcos (pp. 533-554). Cuenca: Ediciones de la Universidad de Castilla-La Mancha.

Santos Santos, J. F. y Moreno Díaz del Campo, F. J. (2009). Territorio, agua y sociedad. Hacia un modelo de distribución de los molinos harineros en el entorno de Las Tablas de Daimiel. En C. Barba Ruedas, J. F. Sánchez Ruiz y A. Alonso López (Coords.), V Congreso Internacional de Molinología. Actas (pp. 729-739). Toledo, España: Junta de Comunidades de Castilla-La Mancha.

Solano Ruiz, E. (1976). La Orden de Calatrava en el siglo XV. Los señoríos castellanos de la orden al final de la Eda Media. Sevilla: Universidad de Sevilla. 